\title{
AURORAL ZONE EFFECTS ON HYDROGEN GEOCORONA STRUCTURE AND VARIABILITY
}

\author{
T. E. MOORE, A. P. BIDDLE and J. H. WAITE, Jr. \\ Space Science I aboratory, NASA/Marshall Space Flight Center, \\ Huntsville, AL 35812, U.S.A. \\ and \\ T. L. KILLEEN \\ University of Michigan, Ann Arbor, Michigan, U.S.A.
}

(Received 16 Octobern1984)

\begin{abstract}
The instantaneous structure of planetary exospheres is determined by the time history of energy dissipation, chemical, and transport processes operative during a prior time interval set by intrinsic atmospheric time scales. The complex combination of diurnal and magnetospheric activity modulations imposed on the Earth's upper atmosphere no doubt produce an equally complex response, especially in hydrogen, which escapes continuously at exospheric temperatures. Vidal-Madjar and Thomas (1978) have discussed some of the persistent large scale structure which is evident in satellite ultraviolet observations of hydrogen, noting in particular a depletion at high latitudes which is further discussed by Thomas and VidalMadjar (1978). The latter authors discussed various causes of the $\mathrm{H}$ density depletion, including local neutral temperature enhancements and enhanced escape rates due to polar wind $\mathrm{H}^{+}$plasma flow or high latitude ion heating followed by charge exchange. We have reexamined the enhancement of neutral escape by plasma effects including the recently observed phenomenon of low altitude transverse ion acceleration. We find that, while significant fluxes of neutral $H$ should be produced by this phenomenon in the auroral zone, this process is probably insufficient to account for the observed polar depletion. Instead, the recent exospheric temperature measurements from the Dynamics Explorer-2 spacecraft suggest that neutral heating in and near the high latitude cusp may be the major contributor to depleted atomic hydrogen densities at high latitudes.
\end{abstract}

\section{INTRODUCTION}

The distribution of hydrogen in the terrestrial exosphere is of considerable intrinsic interest (see reviews by Tinsley, 1974; Fahr and Shizgal, 1983). In addition, the altitude profilc of hydrogen densitics is of some importance in the transport of plasma from the ionosphere into the plasmasphere, inner magnetosphere, and geomagnetic tail. Moore $(1980,1984)$ has argued that the composition of the outflowing plasma is controlled by an interaction between acceleration and chemical processes in the topside ionosphere. The densities of neutral $\mathbf{H}$ and $\mathbf{O}$ atoms are of great importance in the chemical aspect of the interaction.

The global hydrogen budget has been discussed by Vidal-Madjar and Thomas (1978) and Thomas and Vidal-Madjar (1978). Those authors reported evidence for a persistent depletion of atomic hydrogen above 500 $\mathrm{km}$ altitude at auroral and polar cap latitudes. A similar polar depletion has been reported by Emerich and Cazes (1977), based upon an independent data set. Thomas and Vidal-Madjar (1978) computed the enhancement in polar-neutral temperature which would be required to produce equilibrium in lateral transport; and alternatively, the radial flux of hydrogen required to balance the lateral transport in the absence of a temperature gradient. They found that the polar wind flux of hydrogen ions is insufficient to account for the observed density depletion, and asserted that the required neutral temperature increases $(400-600 \mathrm{~K})$ were probably fictitious. They concluded that either there was some error in the interpretation of ultraviolet observations or some unrecognized ion-neutral coupling process was present in the high latitude exosphere. Hodges et al. (1981) subsequently suggested that the use of realistic hydrogen velocity distributions (rather than complete Maxwellians) in the radiative transfer calculations might eliminate or reduce the inferred depletion. However, this possibility does not appear to have been pursued to the point of providing an alternative explanation of the apparent polar depletion.

One possibility discussed by Thomas and VidalMadjar (1978) is the enhancement of $\mathrm{H}$ escape by charge exchange of fast ions with neutral $\mathrm{H}$, resulting in a fast neutral and a slow ion. This effect has been explored with application to the low latitude plasmaspheric regions of diffusive equilibrium by a 
variety of authors (see, e.g. Fahr and Shizgal, 1983; and references in Thomas and Vidal-Madjar, 1978). It has been fairly conclusively demonstrated that this effect leads to a significant enhancement of the neutral hydrogen escape flux from the Earth, which helps to account for the apparent discrepancy between the thermal evaporation flux and the mesospheric hydrogen flux (Hunten and Strobel, 1974; Liu and Donahue, 1974; Tinsley, 1978). This effect has also been explored for the ionosphere of Venus by Hodges and Tinsley (1981). This effect was not evaluated for polar wind conditions by Thomas and Vidal-Madjar, though they asserted that it would be unlikely to contribute significantly due to the depressed $\mathrm{H}^{+}$densities in regions of outward flow.

Recently, another type of ion escape flow has been found to occur frequently in the terrestrial auroral zones, involving transverse heating of the ambient ion population at altitudes as low as $500 \mathrm{~km}$ (see e.g., Klumpar, 1979; Yau et al., 1983; Lockwood, 1983). The signature of this acceleration or heating is the presence of substantial fluxes of ions having an angular distribution which is strongly peaked between $90^{\circ}$ and $180^{\circ}$ pitch angle relative to the local magnetic field, where $0^{\circ}$ pitch angle is taken as downward into the ionosphere. Such fluxes are often observable up to energies of several $100 \mathrm{eV}$ and decrease monotonically with energy above the instrumental low-energy limit (generally of the order of $10 \mathrm{eV}$ ). This signature is interpreted as being gyrotropic with respect to the magnetic field, so that the flux peaks on the surface of a cone whose half-angle varies from 90 to $180^{\circ}$ with respect to the downward direction along the local magnetic field. The terms "transversely accelerated ions" and "ion conics" have been frequently used to label this signature, which is thought to result from preferential heating perpendicular to the magnetic field at some altitude; with subsequent upward flow under the influence of the magnetic mirror force leading to evolution of the "conic" angle from 90 toward $180^{\circ}$.

Since this heating occurs at altitudes where neutrals are plentiful, charge exchange induced escape may well become a significant effect even at high latitudes where the heated ions escape rapidly. In view of the importance of polar hydrogen escape in understanding the observations of hydrogen densities, we have explored quantitatively the charge exchange induced hydrogen escape associated with polar wind and transversely accelerated ion flows, and compared our results with the previously explored case based on plasmaspheric diffusive equilibrium.

We have also examined the question of high latitude neutral atmosphere temperature enhancements as a basis for accounting for the observed hydrogen depletion at high latitude. In particular, recent measurements of exospheric temperatures show that polar cap temperature enhancements are produced by energetic processes in the dayside cusp and auroral zone (Killeen et al., 1984; Hays et al., 1984).

\section{CalCUlation TECHNIQUE}

Our estimates are based on a generalization of the expression used by Maher and Tinsley (1977) to account for anisotropic flowing ion distributions. The flux is estimated by integrating the volume production rate throughout a column of unit cross section extending from the exobase up to an altitude where little contribution is found.

$$
\begin{aligned}
\Phi=\int_{R_{0}}^{R_{m}} \mathrm{~d} R\left(\frac{R}{R_{0}}\right)^{2} & \int_{\operatorname{esc} \mathbf{v}_{i}} \mathrm{~d}^{3} \mathbf{v}_{i} f_{i}\left(\mathbf{v}_{i}\right) \int_{\mathrm{all} \mathbf{v}_{n}} \\
& \times \mathrm{d}^{3} \mathbf{v}_{n} f_{n}\left(\mathbf{v}_{n}\right) \sigma\left(\left|\mathbf{v}_{i}-\mathbf{v}_{n}\right|\right)\left(\left|\mathbf{v}_{i}-\mathbf{v}_{n}\right|\right) .
\end{aligned}
$$

In practice, we have used $11,000 \mathrm{~km}$ as our upper altitude limit, the critical heights given by Banks and Kockarts (1973) as lower altitude limits, and all fluxes have been normalized to $1000 \mathrm{~km}$ altitude. Since the cross-section is only weakly velocity dependent, useful estimates may be obtained without numerical velocity integration by using a value of the cross-section appropriate to the temperatures of interest $\left(7 \times 10^{-18}\right.$ $\mathrm{m}^{2}$ for temperatures of a few $\mathrm{eV}$ or less).

$$
\begin{aligned}
\Phi=\int_{R_{0}}^{R_{m}} \mathrm{~d} R\left(\frac{R}{R_{0}}\right)^{2} M N_{i} \sigma\left(u_{i}^{2}\right. & +\frac{1}{3} v_{t i \|}^{2} \\
& \left.+\frac{2}{3} v_{t i \perp}^{2}+v_{t n}^{2}\right)^{1 / 2} N_{n} .
\end{aligned}
$$

Here $N_{i}$ is the ion number density, $u_{i}$ is the ion flow velocity, $v_{t i \|}$ is the ion parallel thermal velocity, $v_{t i \perp}$ is the ion perpendicular thermal velocity, $v_{t n}$ is the neutral thermal velocity, $N_{n}$ is the neutral number density, $M$ is the fraction of the ion distribution, and hence the fraction of the produced neutral distribution, having escape velocity in the upgoing hemisphere of velocity space. For a bi-Maxwellian distribution, we have for $M$ an expression which must be evaluated numerically :

$$
\begin{aligned}
M=\int_{\substack{|v|>v_{a} \\
v_{\|}>0}} \mathrm{~d}^{3} v\left(\frac{m_{i}}{2 \pi k T_{i \|}}\right)^{1 / 2}\left(\frac{m_{i}}{2 \pi k T_{i}}\right) \\
\quad \times \exp \left[-\frac{m_{i}\left(v_{i \|}-u_{i}\right)^{2}}{2 k T_{i \|}}\right] \exp \left[-\frac{m_{i} v_{i \perp}^{2}}{2 k T_{i \perp}}\right]
\end{aligned}
$$

where we have used $T_{i \|}=m_{i} v_{t i \|}^{2} / 2$ and $T_{i \perp}=m_{i} v_{t i \perp}^{2} / 2$.

\section{INPUTS}

We have included charge exchange reactions with both neutral hydrogen and neutral oxygen. MSIS 
(Hedin, 1983) neutral atmospheres were used to describe the $\mathrm{H}$ and $\mathrm{O}$ density profiles for three cases representing solar minimum, maximum, and "typical" conditions. The solar minimum case had relatively high hydrogen densities and low oxygen densities compared with the solar maximum case. Exospheric temperatures of 753,1063 and $1510 \mathrm{~K}$ characterized the minimum, typical, and maximum cases, respectively. It should be noted that the observations of Thomas and VidalMadjar (1978) were from a solar maximum period, so that this case is of primary interest for comparison.

The first case we have considered is similar to that studied by Maher and Tinsley (1977), i.e., a diffusive equilibrium, plasmaspheric hydrogen altitude distribution. In this case, we have used the $\mathrm{H}^{+}$ion density and temperature profiles for diffusive equilibrium from the work of Raitt and Schunk (1983), their Fig. 3, trace (a). The $\mathrm{H}^{+}$ion flux in this model is zero. We find results similar to those of Maher and Tinsley (1977), though slight differences appear due to differences in the topside ionosphere model and MSIS neutral models.

For the case of a polar wind $\mathrm{H}^{+}$flow, we combined the low altitude model shown as trace (g) of Fig. 3 of Raitt and Schunk (1983), with the high altitude model shown in Fig. 1 of Schunk and Watkins (1982) (curves labeled 1). This high altitude model includes temperature anisotropies which were retained in our calculation. The input parameters derived from these models were thus density, velocity, and parallel and perpendicular temperatures. The $\mathrm{H}^{+}$escape flux normalized to $1000 \mathrm{~km}$ altitude in these models is 1.2 $\times 10^{12} \mathrm{~m}^{-2} \mathrm{~s}^{-1}$. Strictly speaking, these models are applicable only for the neutral atmosphere for which they were calculated, which most closely resembles our MSIS "typical" case. However, we feel that our calculation should adequately illustrate trends in fast neutral production which would be found in selfconsistent ion and neutral flow calculations.

For the case of transversely accelerated $\mathrm{H}^{+}$ions, we have failed to find a truly satisfactory model of the outflow which results when large perpendicular temperature enhancements are introduced at altitudes above $500 \mathrm{~km}$, as has been observed in the auroral zone. In order to derive a crude estimate, we have assumed a bi-Maxwellian distribution at and below $1000 \mathrm{~km}$ altitude having a density of $140 \mathrm{~cm}^{-3}$, parallel temperature of $0.3 \mathrm{eV}$, and perpendicular temperature of $3 \mathrm{cV}$. This distribution is assumed to carry an upward flux corresponding to the flux in the upgoing hemisphere of velocity space, i.e., to have an upward bulk flow equal to the parallel thermal speed. The result is an upward flux of $1 \times 10^{12} \mathrm{~m}^{-2} \mathrm{~s}^{-1}$ normalized to $1000 \mathrm{~km}$. Observed distributions of transversely accelerated ions have non-Maxwellian perpendicular velocity distributions, but their temperature moments are similar to our assumed Maxwellian temperature.

The $\mathrm{H}^{+}$distribution is assumed to continuously relax into a biMaxwellian form. The perpendicular temperature is assumed to fall with altitude as the magnetic field strength, while the parallel temperature rises correspondingly to conserve energy and the first adiabatic invariant. The parallel flow speed is assumed to equal the parallel thermal speed as it rises with altitude. The density is adjusted so as to conserve flux. This procedure effectively ignores the ambipolar electrostatic field and gravity, but does account for the magnetic mirror force. Since hydrogen ions also gain additional energy from the sum of these additional forces, this procedure will slightly overestimate densities at high altitude. If oxygen ions are transversely accelerated, these other forces tend to decelerate and confine them, completely invalidating this procedure. For this reason, we have confined our attention to the production of fast neutral hydrogen. We have set the lower boundary for this case at the critical height or 500 $\mathrm{km}$, whichever is higher.

\section{RESULTS}

Our main results are shown in the matrix of plots given in Fig. 1. In this figure, each plot shows the escaping neutral $\mathrm{H}$ production rate as a function of altitude over the range from the critical altitude (except in the transverse acceleration case, see below) up to $11,000 \mathrm{~km}$. Each column contains plots for a particular level of solar activity, i.e., a particular neutral atmosphere model. The first column contains the solar maximum case of primary interest in relation to the results of Thomas and Vidal-Madjar (1978). Each row contains plots for a particular ion outllow model.

Beginning with the top row, corresponding to diffusive equilibrium with no $\mathrm{H}^{+}$ion flow, we find that substantial fluxes of escaping neutral $\mathrm{H}$ are indicated, of the order of $1 \times 10^{12} \mathrm{~m}^{-2} \mathrm{~s}^{-1}$. These neutral hydrogen fluxes are comparable to the proton flux theoretically and observationally associated with polar wind flow outside the plasmasphere. Proceeding from left to right across the top row, we find increasing fast hydrogen production from $\mathrm{H}$ charge exchange (at high altitudes) and a decreasing contribution from $O$ charge exchange (at lower altitudes), as the neutral atmosphere becomes cooler under conditions of lower solar activity.

The middle row, corresponding to a "typical" polar wind flow, produces significantly lower fluxes of neutral $H$ escape, these fluxes being limited to a fraction of the order of $10 \%$ of the $\mathrm{H}^{+}$ion flux in this case. It is clear that the acceleration and consequent density drop has produced a transition from neutral dominated outflow 


\section{PRODUCTION OF ESCAPING NEUTRAL HYDROGEN FROM FAST $H^{+}$BY CHARGE EXCHANGE}
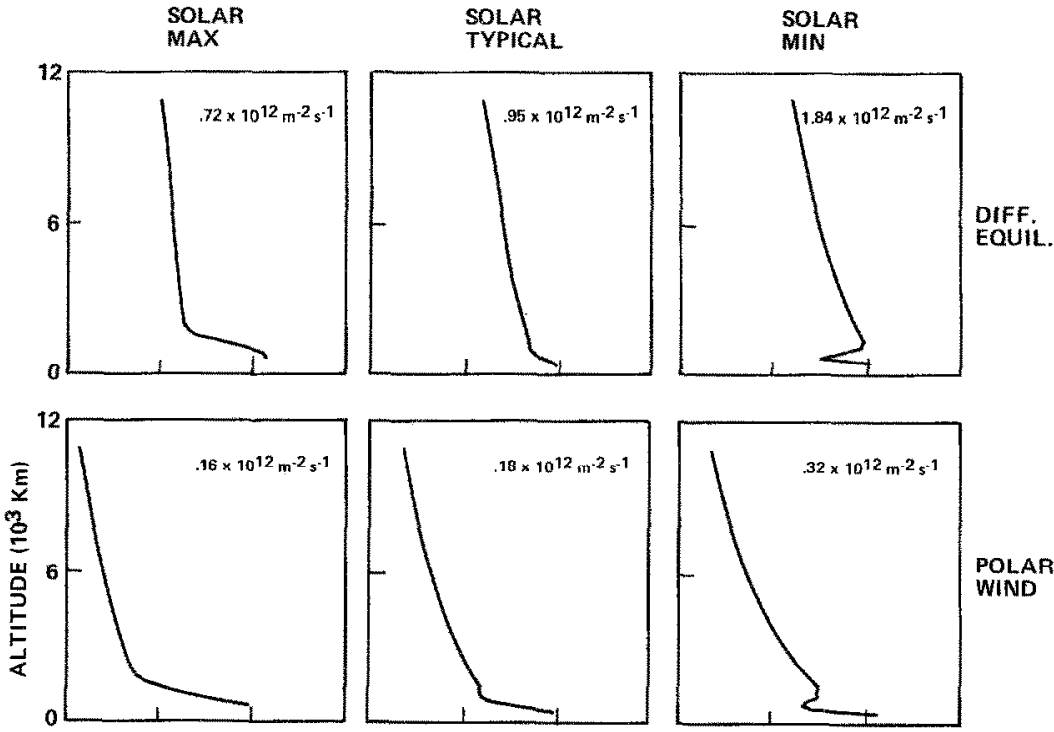

POLAR

WIND

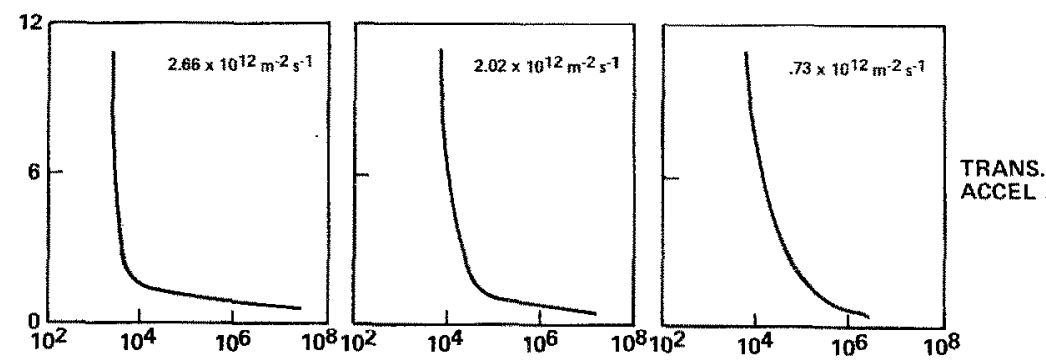

FAST NEUTRAL PRODUCTION $\left(\mathrm{m}^{-3} \mathrm{~s}^{-1}\right)$

Fig. 1. Altitude profiles of PRODUCTION RATES For ESCAPING NEUTRAL HYDROGEN ATOMS BY CHARGe EXCHANGE BETWEEN VARIOUS MODELS OF TOPSIDE HYDROGEN ION DISTRIBUTIONS AND MSIS MODELS OF THE NEUTRAL HYDROGEN AND OXYGEN ATMOSPHERES.

Height integrated production rates give the indicated fluxes of neutral $\mathrm{H}$ as indicated on each plot. See text for further discussion.

to ion dominated outflow, A possible exception is found under solar minimum conditions, when the enhanced hydrogen density leads to more escape production around $1250 \mathrm{~km}$.

The bottom row corresponds to the neutral $\mathrm{H}$ escape produced by our crude model of transverse $\mathrm{H}^{+}$heating at low altitude. It may be seen that the transverse heating has enhanced the escaping $\mathrm{H}$ production at all heights in comparison with the polar wind case. Neutral $O$ charge exchange at low altitudes becomes a major contributor in the solar maximum case. However, even in the solar minimum case, it is clear that transverse heating produces escaping $\mathrm{H}$ fluxes which can be on the order of the $\mathrm{H}^{+}$ion flux, when polar wind flows would produce escaping $\mathrm{H}$ fluxes which are much smaller.

\section{TEMPERATURE OBSERVATTONS}

Recent measurements of polar cap exospheric temperatures obtained by the Fabry-Perot Interferometer (FPI) on the Dynamics Explorer-2 (DE-2) satellite suggest that the apparent decrease in atomic hydrogen density at high latitudes may be explained by 
neutral heating. This possibility, although it was discussed by Thomas and Vidal-Madjar (1978), was dismissed at the time due to inconsistencies in the existing satellite data.

Neutral temperature data from the Fabry-Perot Interferometer (FPI) on DE-2 are shown in Fig. 2. The temperatures indicated by the shading and crosshatching are derived from measurements of the width of the $630.0 \mathrm{~nm}$ emission line of oxygen. The data represent mean oxygen temperatures accumulated from approx. 50 days of data. The sectors which are blank indicate regions not sampled during the data interval because of orbital geometry. These data suggest that the high latitude neutral temperature enhancement required to explain the high latitude $\mathbf{H}$ density depletion may in fact be present. The winter polar cap (Fig. 2 top) is on average $200-300 \mathrm{~K}$ hotter than the auroral zone and midlatitude regions near dusk local time, with polar cap temperatures reaching $1600 \mathrm{~K}$. This somewhat surprising gradient between the polar cap and the auroral zone has been reported earlier by the $D E / F P I$ investigators (Hays et al., 1984). In the absence of a high-latitude heat source, the winter polar cap would be the expected site of a temperature minimum. On the dawn side, the polcward temperature gradient is not as obvious, largely due to the presence of sectors showing very high temperatures at quite low latitudes between 04.00 and 05.00 L.T. The hot spot at $40-50^{\circ}$ latitude is of low statistical significance, since it arises from essentially a single orbital pass, and is not representative of mean conditions. The hot spot indicated at auroral latitudes in this local time sector is more meaningful but is probably associated with transient heating events produced in the dawn sector auroral electrojet. In order to maintain zero net lateral transport of atomic hydrogen in the high latitude exosphere, temperatures in the winter hemisphere that vary from $1400 \mathrm{~K}$ in the polar cap to $1100 \mathrm{~K}$ in the auroral zone (see Fig. 2) require a corresponding polar cap density depletion.

In the summer polar cap (Fig. 2 bottom), although the temperature contrast with surrounding auroral zone and mid-latitude regions is reduced due to high latitude e.u.v. heating, zero net lateral transport would still require polar cap atomic hydrogen density depletions. Temperatures of over $2000 \mathrm{~K}$ have been observed during active magnetic conditions, and transient responses may also be important during such periods. Much of the polar cap heating appears to result from heat advected from the region of the high latitude cusp (Hays et al., 1984). Evidence for strong heat sources in the cusp region includes significant changes in neutral composition in response to geomagnetic activity (Killeen et al., 1985; Waite, et al., 1985).

\section{DISCUSSION AND CONCLUSIONS}

Observed high latitude depletions $(30-40 \%)$ of exospheric atomic hydrogen densities require either (1) an escape rate enhancement over and above normal thermal cscapc $\left(10^{13} \mathrm{~m}^{-2} \mathrm{~s}^{-1} \mathrm{vs} 10^{12} \mathrm{~m}^{-2} \mathrm{~s}^{-1}\right)$, or (2) a temperature enhancement sufficient to maintain zero net lateral transport $(300-500 \mathrm{~K})$ (Thomas and VidalMadjar, 1978). We have investigated the question of whether observed polar depletions of the neutral hydrogen exosphere can be accounted for by suprathermal effects associated with ion-neutral charge exchange. A similar effect at lower latitudes is believed to be a significant, even dominant contributor to the global hydrogen escape flux yielding an escape flux of order $1 \times 10^{12} \mathrm{~m}^{-2} \mathrm{~s}^{-1}$.

We find for a lypical polar wind model that, while an appreciable neutral escape flux is produced, the bulk of the hydrogen escape is in ionized form. The $\mathrm{H}^{+}$ion flux is comparable to the low latitude charge exchange induced escape flux for hydrogen while the polar wind charge exchange induced neutral $\mathrm{H}$ flux is on the order of $15 \%$ of the ion flux. There is an indication in our results that solar minimum conditions may produce slightly larger neutral $\mathrm{H}$ escape in the polar wind because charge exchange on neutral $\mathrm{H}$ becomes increasingly more important.

Transverse heating of $\mathbf{H}^{\prime}$ ions at altitudes as low as $500 \mathrm{~km}$ produces a significant enhancement of escaping neutral production, which becomes substantial for solar maximum conditions due to enhanced neutral $O$ densities. In this case fluxes two to three times the nominal escape flux due to polar wind ion flow or plasmaspheric charge exchange are indicated. This enhancement is in addition to and separate from any neutral heating associated with Joule dissipation or electron heat flux.

Two outcomes of fast neutral production by charge exchange exist, neutral escape and heating of the neutral atmosphere. It should be pointed out that many of the fast neutrals produced are not free to escape due to the magnitude and direction of their velocity vectors. Some bulk heating will occur as these fast neutrals randomize their energy below the baropause or critical height. Rough estimates indicate that this energy source is small compared with competing effects, however.

We thus envisage comparable hydrogen escape rates in the polar cap (due mainly to $\mathrm{H}^{+}$escape) and within the plasmasphere (due mainly to neutral $\mathrm{H}$ escape), with a region of enhanced escape (by a factor not exceeding 2-3) within the auroral oval and polar cusp due to transverse acceleration of the $\mathrm{H}^{+}$flow. Note that this scenario does not offer a clear solution to the 


\section{DE-2 FPI TEMPERATURES (K) MEAN}

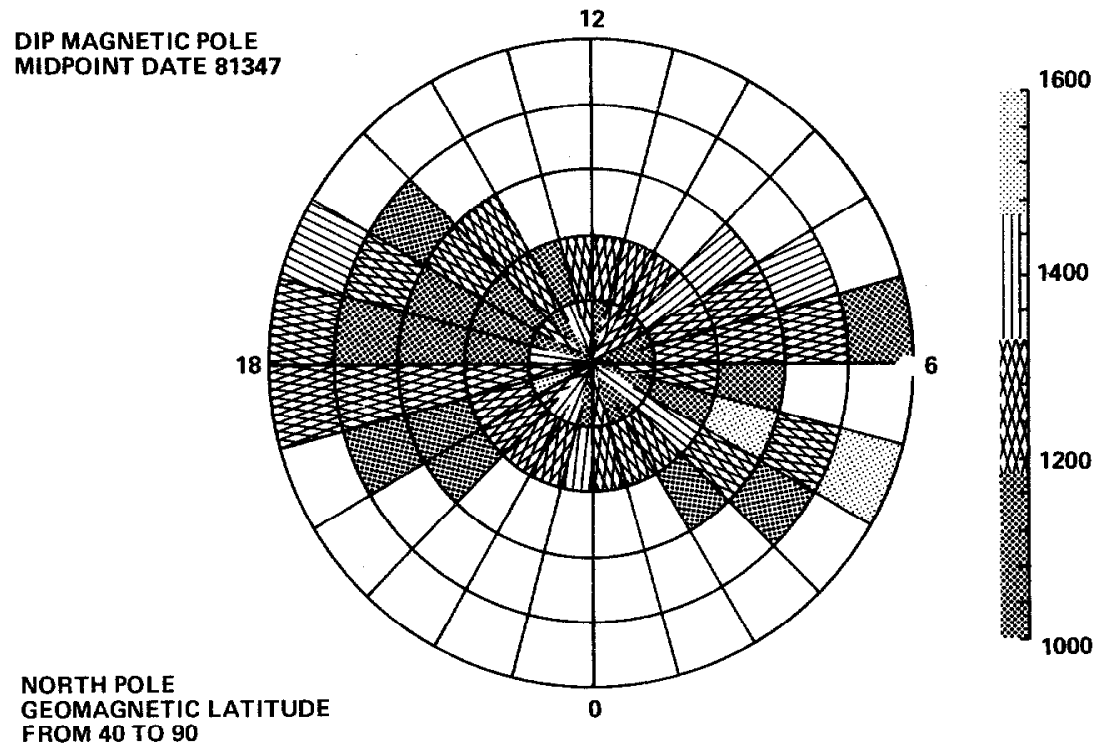

DE-2 FPI TEMPERATURES (K) MEAN

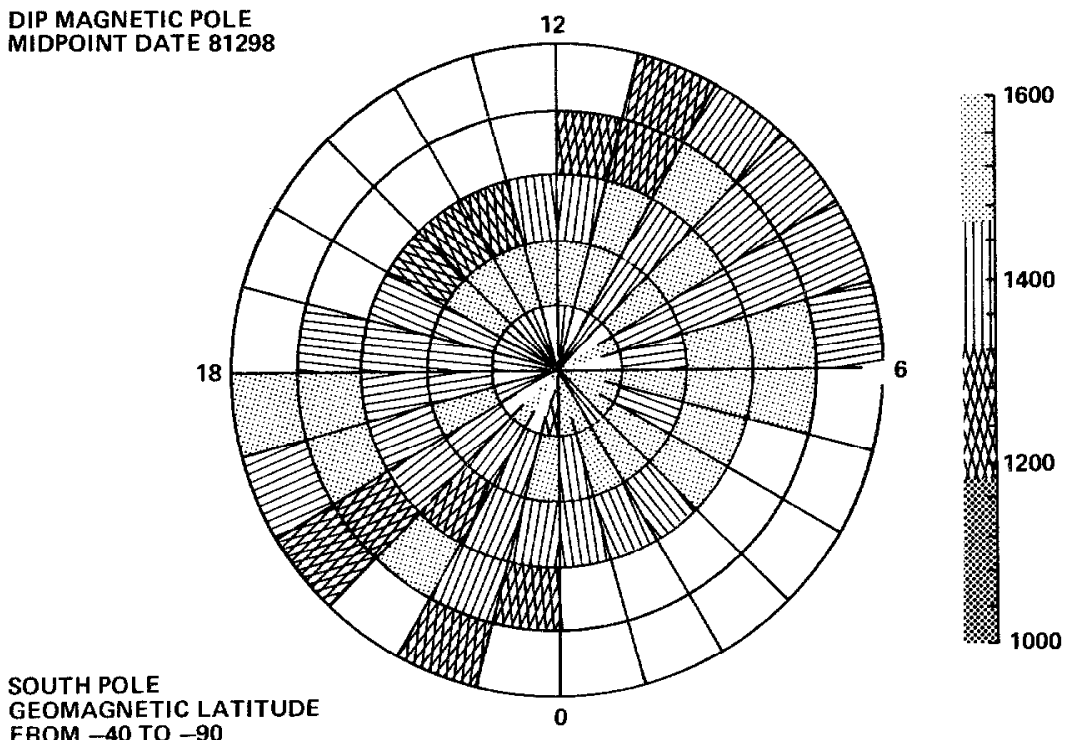

Fig. 2. Dynamics Explorer MEAsurements of NeUtral atmosphere temPerature from the Fabry-Perot INTERFEROMETER.

The upper panel displays data from the winter (North) polar cap while the lower panel displays corresponding data from the summer (South) polar cap. Blank sectors indicate a lack of available data. 
problem of reduced neutral hydrogen densities over the polar cap, since that would require an order of magnitude flux enhancement over the entire polar cap. We conclude that the known superthermal effects, including charge exchange of auroral zone transversely accelerated ions, are insufficient to account for large scale polar cap depletions, though they do make an appreciable contribution.

In view of the temperature enhancements shown in Fig. 2, which appear marginally adequate to support the observed density depletions, it seems possible that the observed latitudinal structure of the hydrogen exosphere is mainly determined by the neutral heating in the dayside cusp region. Auroral zone heating of a thermal nature (Joule heating, energetic ion and electron heat flux) may make a contribution to the polar $\mathbf{H}$ depletion, as well. In either case, the heat added to the neutral atmosphere in the auroral zone will be convectively distributed throughout the high latitude region due to neutral circulation driven by plasma motions induced by the solar wind (Hays et al., 1984).

This leads us to suggest a global scenario in which the subauroral exospheric $\mathrm{H}$ density is controlled by the general level of solar activity through variations in the solar c.u.v. brightness. The polar depletion feature is in turn controlled by magnetospheric convection and the rate of energy deposition into the cusp and auroral neutral thermosphere. Therefore, larger temperature contrasts between polar and subauroral latitudes and correspondingly greater exospheric hydrogen density depletions would be expected under solar minimum and winter conditions than during solar maximum and summer conditions. We are not aware of any ultraviolet observations of neutral hydrogen during solar minimum, with which to test this suggestion. The most recent MSIS model (Hedin, 1983) already incorporales a measure of polar temperature enhancement, and the existing literature on geocoronal hydrogen appears to demand such adjustments.

Acknowledgements-We would like to acknowledge the encouragement provided by A. J. Dessler. This research was supported by the NASA Dynamics Explorer program.

\section{REFERENCES}

Banks, P. M. and Kockarts, G. (1973) Aeronomy. Academic Press, New York.

Emerich, C. and Cazes, S. (1977) Local perturbations of the atomic hydrogen density distribution near the exobase, inferred from D2A Lyman $\alpha$ Airglow Measurements. Geophys. Res. Lett. 4, 523.
Fahr, H. J. and Shizgal, B. (1983) Modern exospheric theories and their observational relevance. Rev. Geophys. Space Phys. 21, 75.

Hays, P. B., Killeen, T. L., Spencer, N. W., Wharton, L. E., Roble, R. G., Emery, B. A., Fuller-Rowell, T. J., Rees, D., Frank, L. A. and Craven, J. D. (1984) Observations of the dynamics of the polar thermosphere. J. geophys. Res. 89 , 5597.

Hedin, A. E. (1983) A revised thermospheric model based on mass spectrometer and incoherent scatter data : MSIS-83. $J$. geophys. Res. 88, 10170.

Hodges, R. R., Jr. and Tinsley, B. A. (1981) Charge exchange in the venus ionosphere as the source of the hot exospheric hydrogen. J. geophys. Kes. 86, $/ 649$.

Hodges, R. R., Rohrbaugh, R. P. and Tinsley, B. A. (1981) The effect of the charge exchange source on the velocity and "temperature" distributions and their anisotropies in the Earth's exosphere. J. geophys. Res. 86, 6917.

Hunten, D. M. and Strobel, D. F. (1974) Production and escape of terrestrial hydrogen. J. Atmos. Sci. 31, 305.

Killeen, T. L., Hays, P. B. and Gell, D. A. (1985) Direct measurements of exospheric temperatures in the polar region. J. geophys. Res. (in press).

Klumpar, D. M. (1979) Transversely accelerated ions: an ionospheric source of hot magnetospheric ions. J. geophys. Res. 84, 4229.

Liu, S. C. and Donahuc, T. M. (1974) Mesospheric hydrogen related to exospheric escape mechanisms. J. Atmos. Sci. 31, 1466.

Lockwood, M. (1983) Thermospheric control of the auroral source of $\mathrm{O}^{+}$ions for the magnetosphere. J. geophys. Res. 89, 301.

Maher, L. J. and Tinsley, B. A. (1977) Atomic hydrogen escape rate due to charge exchange with hot plasmaspheric ions. $J$. geophys. Res. 82, 689 .

Moore, T. E. (1980) Modulation of terrestrial ion escape flux composition. $J$. geophys. Res. 85, 2011.

Moore, T. E. (1984) Superthermal ionospheric outflows. Rev. Geophys. Space Phys. 22, 264.

Raitt, W. J. and Schunk, R. W. (1983) Composition and characteristics of the polar wind, in Energetic Ion Composition in the Earth's Magnetosphere (Edited by Johnson, R. G.). Terra, Tokyo.

Schunk, R. W. and Watkins, D. S. (1982) Proton anisotropy in the polar wind. $J$. geophys. Res. 87, 171 .

Thomas, G. E. and Vidal-Madjar, A. (1978) Latitude variations of exospheric hydrogen and the polar wind. Planet. Space Sci. 26, 873.

Tinsley, B. A. (1974) Hydrogen in the upper atmosphere. Fund. Cosmic Phys. 1, 201.

Tinsley, B. A. (1978) Effects of charge exchange involving $\mathbf{H}$ and $\mathrm{H}^{+}$in the upper atmosphere. Planet. Space Sci. 26, 847.

Vidal-Madjar, A. and Thomas, G. E. (1978) The terrestrial hydrogen problem. Planet. Space Sci. 26, 863.

Waite, J. H., Jr., Nagai, T., Johnson, J. F. E., Chappell, C. R., Burch, J. L., Killeen, T. L., Hays, P. B., Carignan, G. R., Peterson, W. K. and Shelley, E. G. (1985) Escupe of suprathermal $\mathrm{O}^{+}$ions in the polar cap. J. geophys. Res. (in press).

Yau, A. W., Whalen, B. A., McNamara, A. G., Kellogg, P. J. and Bernstein, W. (1983) Particle and wave observations of low-altitude ionosphericion acceleration events. J.geophys. Res. 88, 341. 